\title{
OBSERVATIONS ON NEW DEVELOPMENTS IN COMPOSABILITY AND MULTI-RESOLUTION MODELING
}

\author{
Paul K. Davis \\ RAND Corporation \\ Santa Monica, CA 90407, U.S.A.
}

\author{
Andreas Tolk \\ Engineering Management \& Systems Engineering \\ Old Dominion University \\ Norfolk, VA 23529, U.S.A.
}

\begin{abstract}
MRM (MRM) and Composability are two of the most challenging topics in M\&S. They are also related. In this paper, which was written to set the stage for conference discussion of related papers, we discuss how addressing the MRM challenge is sometimes a necessary - although not sufficient - step towards solving the composability challenge. This paper summarizes recent developments in theory drawing distinctions among issues of syntax, semantics, pragmatics, assumptions, and validity. The paper then discusses how technology for ontology development may be useful in improving both composability and MRM. Two examples illustrate how some of the issues arise. One involves a large analytic war gaming system from the past; the other involves current counter-terrorism modeling in which many of the complications are due to the socialscience nature of the problem area.
\end{abstract}

\section{INTRODUCTION}

The purpose of this paper is to give an update on recent developments in the research domains of composability, ontology, and MRM (MRM). We also draw relationships to the Software Engineering Body of Knowledge effort (SWEBOK project).

\subsection{Background}

Composability has been identified for some years as a key and difficult challenge for M\&S. The challenge cannot be avoided, because some degree of composability is necessary in real-world problems. The manifold reasons include:

- Software is expensive and it is economically desirable to reuse established software whenever possible.

- Even if economics were not a factor, achieving reliability in complex and sophisticated systems depends on not reworking solved problems needlessly.
- It is highly desirable in some applications to be able to use alternative submodels developed by different people with different perspectives and ideas, sometimes as part of distributed developments. Often, this will require composition, which should be as painless as possible (i.e., it should require relatively little new programming).

- Commercial information technology (IT) systems and military command and control (C2) systems are moving toward service-oriented architectures (SOA). Instead of specifying all requirements for a system before it is build and implementing the system with hardwired component functions, functionality is encapsulated in services that can drawn upon as needed, a kind of composition.

- Many important applications, such as models and software for Homeland Security and Disaster Relief organizations, require support and reasonably good integration of heterogeneous processes and underlying IT infrastructures.

In summary, economic, technical, and operational considerations call for a considerable degree of composability, as in the use of composable services.

\subsection{Issues in Composability}

This said, recent theoretical work and the experience of practitioners have moderated the demand for "pure composability," which is now seen to be unreasonable (Davis and Anderson 2005). Strict plug-in/plug-out is unlikely to be valid for models, except in special cases, because of substantive subtleties about the component models and the assumptions that underlie them. It is much more feasible to design models in a fashion that will allow subsequent composition in short amounts of time-e.g., hours or weeks, rather than months or years. Time will typically be required for reviewing and adapting the would-be components, supplementing them, and tailoring the assembly, but when viewed from afar, the result can look much more 
like "composition" than major reprogramming, much less starting from scratch. In the special cases, which will probably involve single-project system engineering rather than more general collaboration, plug-in, plug-out may be achieved or approximated.

Some of the key enablers of composability have been much discussed over the last decade. Several frameworks have been proposed, most of them involving layered models. In discussing composability challenges for models and simulations, it has proven useful to explicitly distinguish among five issues identified and embraced in a recent national-academy report (NRC 2006). These issues involve syntax, semantics, pragmatics, assumptions, and validity. It has been common, especially in work by computer scientists, to consider the last four of these to be part of "semantics," but that has had the important effect of obfuscating importantly different classes of problems.

\subsection{Related Issues in MRM}

MRM is very important in analysis-oriented modeling, independent of composability desires (Davis and Bigelow 1998, Davis 2002). It can also be an important enabler of composability. MRM identifies decompositions into components and subcomponents that make sense conceptually and analytically, especially from a top-down perspectivealbeit, one that should be informed by a bottom-up understanding of phenomena. Further, careful MRM addresses the troublesome issue of imperfect decompositions. It recognizes that "clean" decompositions such as those generating pure tree structures are usually valid only as contextdependent approximations. More generally, "everything is connected to everything" and graphical depictions look more like "bushy trees." Although nice hierarchical trees that make it easy to do top-level analysis with only a few variables, and to then zoom into detail selectively where useful, the bushy-tree representation is often a more accurate representation. Thus, finding good approximations to clean up the representation is necessary.

The MRM process of thinking about context-dependent assumptions is not yet used extensively because it requires expertise that is not widely taught and a measure of subject-area theory. Nevertheless, in many ways it is related to thinking about issues of pragmatics, assumptions, and validity. Further, anyone "doing" MRM must address syntactic and semantic issues. This said, there has been only minimal discussion in the prior literature about the overlap of the MRM and composability challenges.

In any case, the same motivations for composability are relevant as motivations of MRM as well. These include: the desire for modularity and re-use, the desire to make use of "services" where that makes sense, and the need to collaborate effectively across boundaries of resolution, disciplinary perspective, and context of use.
Although a full survey would be well beyond the scope of this paper, the following sections identify a number of developments that should be seen as related; they also elaborate on categories of issue that are very useful in improving the quality of enquiry and discussion. Some of the concepts are illustrated with the sketch of a particular recent application, after which the paper ends with some conclusions. We begin by defining composability and requirements for a model to be composable.

\section{COMPOSABILITY}

Composability refers to the ability to select and assemble components in various combinations to satisfy specific user requirements meaningfully (NRC 2006). The NRC report distinguishes composability from interoperability by the requirement that it be readily possible to assemble components differently for different purposes. Interoperability, on the other hand, may be achieved only for a particular configuration, perhaps in an awkward one-time lash-up. To put it differently, composability is associated with modular building blocks. It is also useful to think of composability as a property of the models rather than of the particular programs. These definitions and distinctions draw on suggestions from several recent studies-Petty and Weisel (2003), Davis and Anderson (2003), Page, Briggs, and Tufarolo (2004), and Bartholet et al. (2004). Although by no means universal, the definitions appear to us be sound and useful, and they have benefited from much community-wide discussion and review. A differentlystated but generally consistent way to look at the distinctions was proposed by Page, Briggs, and Tufarolo (2004) and modified in Tolk et al. (2006) based on additional insights derived from Hofmann (2004) and Yilmaz (2004):

- Interoperability deals with the software and implementation details of interoperation, including exchange of data elements based on a common data interpretation, which can be mapped to the levels of syntactic and semantic interoperability. Here we are on the simulation side of M\&S, how the models are actually implemented and executed.

- Composability addresses the alignment of issues on the modeling level. The underlying models are meaningful abstractions of reality used for the conceptualization being implemented by the resulting simulation systems. Composability deals with the Contextualized Introspective of Common Conceptual Models.

In summary, it is convenient to refer to the interoperability of simulations and to the composability of models.

Composability has many facets that need to be addressed; distinguishing among them, and their associated 
challenges, is essential. Many discussions still refer to problems being either syntactic or semantic, but the situation is more complex than is conveyed by such a description. The following paragraphs provide a tutorial and recommend distinctions that need to be made systematically. They deal with syntax, semantics, pragmatics, assumptions, and validity. This view is the one applied in (NRC 2006):

- Syntax: Consistency of syntax means that two models can operate together on the technical level, i.e., the digital output from one can be read as the digital input to the other. Protocols assure syntactical consistency among models to be connected. At the simplest level, a computer model written in a particular language may use submodels represented as functions. The computer model must use certain key words and syntax to signal that what follows is the name of a function and its list of input parameters, followed then by a continuation of the program. Web services are enabled by XML that defines input- and outputparameters. The Object Model Template of the High Level Architecture is another example of rules, or protocols, assuring syntactical compatibility.

- Semantics: Consistency of semantics ensures that data have the same meaning in the sending and receiving model. If some data can flow from Model A to Model B, the semantic question is whether that data is understood by both $\mathrm{A}$ and $\mathrm{B}$ to refer to the same thing (e.g., the number of soldiers in a military unit). To computer scientists, the operational meaning of semantic consistency is often much narrower and computer oriented. Tolk (2003) recommended the use of common reference models to overcome these challenges by mapping information exchange objects to standardized data elements; Tolk et al. (2006) give examples of successful applications. Enabling technologies and solutions are languages based on a standardized common reference model, the standardized Protocol Data Units of the Distributed Interactive Simulation Protocol, or the object model of the Test- and Training Enabling Architecture (TENA).

- Pragmatics: Consistency of meaning is not always straightforward because the same word means different things depending on context. Moreover, key aspects of context may not be explicit. This is the realm of pragmatics. While semantics ensures that the individual data elements are unambiguously identified, pragmatics puts these data into context of how they are used in the model. Hofmann (2004) introduced the ideas of pragmatically associated entities that led to the definition of "composites" or "transactionals" in Tolk et al. (2006). Even if two models agree on the meaning of all the data elements to be exchanged, they still may use these data elements in different contexts, making the exchange of these data during runtime challenging. In military applications, for example, an "attack location" may refer variously to the location of the planned attack, the current location of the attacking unit, the location of the object to be attacked, and so on. Only in the context of a "composite," such as locationof-attacking unit, does the context become unambiguous.

- Assumptions: Even after establishing consistency between models in the way words are used, difficulties continue. The way Model A calculates the data may not be suitable for what B needs. Sometimes this may be a matter of precision or accuracy, but other times it may be considerably more subtle. The datum of "temperature," for example, might refer to a surface temperature, an average temperature over some path into the ocean relevant to a sensor, the ambient air temperature on a battlefield with very hot moving objects, or something else again. To the extent that it's just a matter of clarification about which temperature is meant, that is a matter of pragmatics. However, it may instead involve an assumption, such as the assumption that what a wall sensor measures is characteristic of a large room. In medicine, an example is the level of "bad" cholesterol reported from a blood test: that level is usually an estimate based on a calculation. It is not always accurate.

- Validity: Finally, there is the question of whether the model is correct. A model's assumptions may in fact be wrong, the logic or calculations used may be wrong, or both. Further, a model that is adequately valid in one context may not be valid in another-even if issues of syntax, semantics, pragmatics, and assumptions are taken care of. There are some overlaps here, to be sure. The lack of validity may be due to changes of context that could be regarded as an issue of pragmatics: "Ah, you meant effectiveness for such-and-such as mission in such-and-such an environment! I thought you meant something else." However, the lack of validity may instead be something more mathematical, as when an average quantity is no longer adequately representative.

Compositional validity issues can even be studied at a formal level. Weisel, Petty, and Mielke (2003) have done so and demonstrated difficult issues. Tolk (1999) gives a number of examples in which the composition of valid federates into a 
federation doesn't ensure validity of the final result.

To address various issues mentioned, several frameworks have been proposed (although each other has referred to the issues in somewhat different language). We shall mention only a few of them here, and certainly to not intend to denigrate those not presented.

The first proposed solution distinguishes among conceptual model, implemented model, simulator, and experimental frame. The desirability of distinguishing between a conceptual model and a program representing a particular implementation of that conceptual model has been emphasized for decades by thoughtful scholars such as Zeigler, Praehofer, and Kim (2000). The recommended framework for M\&S establishes entities and their relationships. The entities of the framework are real world referent, experimental frame, model, and simulator. The real world referent is observed in the context of the experimental frame, which specifies all constraints of the observation. The modeling relationship links the referent in the context of the experimental frame with the model. The model is linked via a simulation relationship to the implemented model, which is executed as a simulator. Each entity is formally characterized as a system at an appropriate level of specification within a generic dynamic system. Yilmaz and Oren (2004) use these ideas for a conceptual model for reuse, close to the recommendations given by Davis and Anderson (2003). In addition to this high-level model view, the authors see documentation as a critical supporting element of composability and advocate the potential of a high-level graphical specification system coupled with a detailed specification system, for example the Discrete Event Specification System (DEVS). Besides describing the functionality of the component it is essential that this documentation captures syntactical, semantic, pragmatic, assumptions, and validity issues as discussed before.

Tolk et al. (2006) document a framework based on a common reference model to capture standardized data elements for information exchange in a net-centric Web service based environment, implementing the ideas of modelbased data engineering as documented in Tolk and Diallo (2005). The common reference model is accessed via wrapping Web services. The Web service protocol itself establishes syntactic interoperability as defined earlier in this section.

- The inner core comprises the atomic services. Each standardized data element of the common reference model is represented by one atomic service. These atomic services establish semantic interoperability, as each data element to be exchanged is represented by a well designed set of at least one standardized data element. If the com- mon reference model is a relational model, the content that can be used to populate every table is represented as an atomic service. Atomic services can check the consistency with the standardized data element, such as attribute value ranges, existence of mandatory fields, etc.

- The atomic services are associated within the common reference model. Using these associations, "composites" that ensure that transactions are consistent within the common reference model are defined. Therefore, they are also referred to as "transactionals." These resulting composite services represent the information that can be exchanged reflecting the pragmatic issues. In a relation model, these are valid views representing associated tables. Within composite services, consistency of transactions can be checked and ensured.

- Finally, aggregation, filtering, and transformation processes are applied on top of these composite services to reflect the assumptions of the participating models. This group of services is often referred to as mediation services. They can be configured using the results of model-based data engineering. While the reference model supports all potentially needed information, a filter can be applied to access only those data needed by the application. If the unit of measure is different, transformations are needed. If different levels of resolution are supported, aggregation is needed. The ideas described by Pullen et al. (2004) for Web service interest management can be applied in this context.

For practitioners it should be pointed out that the resulting "cascading" web service structure does not have to be implemented as a series of nested web service calls. Perme et al. (2007) describe an application example of such a family of services.

Finally, the elements of the ontological spectrum as defined by Daconta, Obrst, and Smith (2003) can capture the necessary metadata enabling automation using intelligent agents. Gruber (1993) defines ontologies as formal descriptions used to describe and categorize concepts and the relationships among concepts within a particular knowledge domain. They can be processed by machines or read by domain experts. Current ideas are summarized by Tolk and Turnitsa (2007). This is not the first ontological application for M\&S: Benjamin, Patki, and Mayer (2005) give a more technical view on simulation support (in contrast to the modeling support) for ontology applications. Silver, Lacy, and Miller (2006) use the same means to support the process interaction world view. However, the focus of these and related ontological efforts was on the 


\section{Davis and Tolk}

simulation side, whereas composability needs to focus more on the modeling side of M\&S.

While the technical means are falling into place, there are other issues to solve as well. Spiegel, Reynolds, and Brogan (2005) conducted a small case study to clarify the role that model context plays in simulation composability and reusability. For a simple problem, computing the position and velocity of a falling body, they found that a reasonable formulation of a solution included a surprising number of implicit constraints. This work shows the practical challenges in identifying assumptions and constraints. This said, some thoughtful listing of assumptions by developers, even if telegraphically stated, is far better than none.

In addition to these problems, complexity issues, such as described by Page and Opper (1999) and Bartholet, Brogan, and Reynolds (2005), need to be solved as well: it has been proven, among other challenges, that the component selection problem, given a set of simulation components and a set of objectives, is NP-complete (Weisel, Petty, and Mielke 2003). The task to support composability of services is therefore neither conceptually nor computationally trivial.

One suggestion that we have is that those concerned with developing metadata standards and, more important perhaps, recommended procedures for developing metadata, should give far more emphasis to "substantive" modeling issues such as assumptions. How could key assumptions be economically encapsulated in metadata and subsequently understood, at least to some degree, by intelligent agents? Insights on how to accomplish this need to come from experienced analysts and modelers, not "software folks." For example, the better analysts and modelers have learned over time how to summarize key assumptions in a single page or table. What can be generalized from their experience, both within a subject area and for crosscutting purposes? General solutions are unlikely, but very useful guidelines for developing model metadata are surely feasible.

In summary, even with the proposed frameworks the problem of composability remains challenging, but significant progress has been made even since the report on "Improving the Composability" (Davis and Anderson 2003). Some of these are summarized in NRC (2006). What is still missing is a common conceptual and accepted frame to merge the various contributions into a consistent theory of composability. The next section discusses the potential contributions of MRM and development of ontologies.

\section{MRM AND ONTOLOGIES}

In order to identify potential contributions of ontologies, we will summarize respective aspects of MRM before enumerating selected aspects of ontological research.

\subsection{MRM}

We define MRM as follows (Davis and Bigelow 1992): MRM is (1) building a single model with alternative user modes having different levels of resolution for the same phenomena; (2) building an integrated family of two or more mutually consistent models of the same phenomena at different levels of resolution; or (3) both. Note that in our definition, the user can specify inputs at different levels of detail; this is very different from building a single bottomup model and allowing for displays with different resolutions. A related concept is Multi-resolution, multiperspective, modeling (MRMPM).

MRM is highly relevant to composability. Although it is still rather unusual and only seldom approached systematically, basic concepts have been published and discussed (Davis and Huber 1992, Davis and Hillestad 1993, Reynolds, Natrajan, and Srinivasan 1997, Radharamanan and Wilsey 1999) along with a number of applications in particular studies (e.g., Davis, Bigelow, and McEver 2000).

MRM modeling is an enabler or exploratory analysis (EA) in which one studies a problem throughout the entire assumptions space, rather than assuming a baseline case and some sensitivity around that. This is arguably essential in problems beset by large uncertainties, including deep uncertainty. Such uncertainty is common in strategic planning generally, including in defense-related force planning and acquisition planning. If a model has been designed with an MRM approach, it is possible to do the exploration initially with a lower-resolution (more abstract) version of the model, with perhaps 3-10 parameters covering the entire space. After varying all of those across the ranges of their plausible values simultaneously in exploratory analysis, one (or more) might turn out to be particularly significant. It might then be desirable to zoom into more detailactivating a higher resolution model that calculates what was previously a parameter in terms of lower-level inputs. Exploration at that level may add deeper insight. For example, a top-level exploration might indicate that a comparison of options was being driven by the vector of costs. If a lower-level cost model were activated, explorations might indicate that the principal uncertainty related to the cost of a particular high-risk satellite system. At yet another level of detail, exploration might reveal that the principal factor was the projected cost per pound to deliver payload to orbit.

In discussing uncertainty, it is important to distinguish between structural uncertainty-i.e., is the very form of the model correct? - and parametric uncertainty, i.e., uncertainty about the values of inputs. One may not be sure that the model is correct structurally, but one has a seemingly reasonable theory or a good deal of experience to support it. Or perhaps the model is solid, but the input data for the application are highly uncertain. For the report, the motiva- 
tion was that both of these circumstances are common in strategic planning and policy analysis and that they are also common in military applications such as operations planning, acquisitions planning, and training. No one has a "correct" model of war with all its notorious complications, and, even if such a model existed, it would have large numbers of uncertain inputs.

When Davis and Bigelow wrote their report, they saw these issues as particularly relevant to models. Interestingly, however, many of the same issues arise in the deeper aspects of software development (Bartholet, Brogan, and Reynolds 2004). They also apply to service-oriented architecture in infrastructural environments as currently defined for the Global Information Grid (GIG): when accepting a service or data provided via the GIG infrastructure, the user can never be sure to what degree the service represents his perspective or the data have been obtained with the required accuracy. Recent studies conducted on authoritative data sources within the U.S. Joint Forces Command in the project on Joint Rapid Scenario Generation (JRSG) show that these data can differ significantly. The same results were shown during earlier Joint Warfighter Interoperability Demonstration (JWID) where the different databases of NATO partners in Europe were compared and showed - although all being certified - significant differences in data about the same concepts, such as length of runways of airports, etc. Problems also arise in new areas of operation, such as Homeland Security or Disaster Relief, where collaborators go about using services differently and make different assumptions about what the services provide.

\subsection{Ontological Research}

Naively, it might be assumed that many of the issues raised above could be resolved by ontological work. Much such work considers only resolution and scope when. Turnitsa and Tolk (2007) evaluate ontological techniques in addressing MRM issues in distributed systems; the authors conclude that it is necessary to address scope, resolution, and structure in characterizing models:

- In a slight variation of Gruber's definition (1995), an ontology is defined as a formal specification of a conceptualization. The concepts of an ontology have content and structure and are associated with other concepts.

- The scope is defined as the set of represented concepts. Following the framework for M\&S as defined by Zeigler, Praehofer, and Kim (2000), concepts are used to model world referents in the model. If two models represent the same concepts (which are implemented as simulated entities within the virtual environment, which translates into simulated entities represent concepts of the model within the simulation), they have the same scope.

- The resolution is the level of detail with which the content of the concepts is presented. This was the original domain of MRM. Aggregation and disaggregation are defined in the realm. A special concept is the representation of time.

- The structure represents the morphology of the ontology, which is the macro-morphology (associations of concepts) and the micro-morphology (properties used within the concept).

Even if two models have the same scope and resolution, the attribute characterizing one concept in Model A may characterize a different concept in Model B. Turnitsa and Tolk (2007) give the example of "number world" and "letter world." The same entities at the same resolution are used in both worlds: A1, A2, B1, and B2. However, while in "number world" $\mathrm{A} 1$ and $\mathrm{B} 1$ build one concept, which is the " 1 "-concept, and A2 and B2 build the " 2 "-concept, in letter world A1 and A2 build the "A"-concept, and B1 and B2 the "B"concept. In the military world such examples can be observed when logistical concepts and operational concepts have to be mapped. The logistician using a storage- and transport-centric approach, while the warfighter uses categories shaped by the use of the material in combat.

The traditional way to deal with MRM challenges assumes (or requires) that a "natural decomposition" of a system is possible, resulting in a hierarchical structure of this system that can be used in support of identifying aggregation and disaggregation domain. However, modes are purposeful abstractions of reality and as such different abstractions serving different purposes can be used to describe the same reality. In other words: There is often no single correct way to design an MRM model of a system. A given system has different facets (Zeigler, Praehofer, and Kim 2000) and can be described from different perspectives, much as physics models can have alternative representations. Thus, no single MRM structure will do, at least in some cases (the reason that Davis and Bigelow refers to multi-resolution, multi-perspective modeling or MRMPM). Using ontological means to describe scope, resolution, and structure as recommended by Tolk and Turnitsa (2007) allows not only the configuration of mediation layers as defined before, but may support agent mediated solutions as envisioned in Yilmaz and Tolk (2007): if services can described by metadata regarding their resolution, their scope, their structure, and underlying assumptions, intelligent software agents can support the selection, composition, and orchestration process efficiently. 
In summary, the importance of MRM and MRMPM increases in the light of composable services. Solving the challenge to align different scopes, resolution, and structures represented in services to be composed in support of a common operation is a necessary-although not sufficient-step towards composability. A first step is to document scope, resolution, and structure with a formal specification of this conceptualization. Ontological research needs to more explicitly address these problems, including the issue of multiple perspective.

\section{EXAMPLES}

We give two example of analytic applications involving models that have raised the issues we have talked about above. One is an old example from the 1980s; one is current.

\subsection{Composition Issues in the RAND Strategy Assessment System (RSAS)}

The RAND Strategy Assessment System (RSAS) was developed and used in the 1980s (Davis 1989, Schwabe 1990). It was large (a million lines of code) and included not only a simulation with component models for various theaters of war worldwide, as well as maritime operations and strategic mobility, but also Red, Blue, and Green Agents, which were an unusual type of artificial-intelligence models representing the decision making of the Soviet Union, the United States, and third countries. In addition to political-level models, Red and Blue had "analytic war plans," which represented decision-making by theaterlevel military commanders attempting to follow war plans, but adapting behaviors from time to time at either anticipated branch points or because of special events that occurred. Humans could play instead of the agents at any of the positions. This was very useful in developing the agents' rules and war plans in the first place.

The political-level decision models had multiple levels of optional detail; i.e., they exemplified MRM. The agents could "think" at a very high level of abstraction- deciding, for example, whether to escalate or de-escalate based on factors such as the current level of conflict, the projected outcome of the war if the current war plan were pursued, a simplified model of the adversary, and so on. A factor such as the projected outcome of the war could be estimated simply, calculated with a somewhat more complicated local model, or calculated from more detail by running the simulation itself using assumptions about the adversary's plan as well as the agent's own plan. When operating in this more detailed mode, the level of conflict would be inferred based on the number of nuclear weapons that had been used, the targets on which they had been used, the period over which they had been used, the most recent time of use, and other variables. In a lower- resolution mode, an agent could project outcome using a data that matched rough characterizations of the situation (e.g., current theater-level force ratio) with results of previous simulations in similar cases.

Another feature of the RSAS was that the analytic war plans were composed from discrete components for various building-block operations, such as "pull forces in suchand-such a corps back to a particular river line," which were defined by sets of specific force orders such as "move US Mech. Division X to such and such a position along the corridor in which it was assigned to fight."

Because of this modular approach, it was possible to have many different war plans that assembled with various combinations of the building-block components and values for parameters within them. A human player could create a new war plan rather easily (for the time), either drawing upon the building blocks and adjusting parameter values, or by adding new components. The plans themselves were motivated by real war plans, war plans used in official studies, and war plans developed in live human play.

As a whole, then, the RSAS had many features relevant to this paper. It was highly modular, the version used in a particular case was "composed" by combining or changing parameter values in those modules, and many of the RSAS models could be used at any of several levels of resolution (i.e., higher levels of detail could be "turned off or on").

It might seem from our description that the RSAS was a composable system, but in the terminology we use in this paper, it was not. It was instead designed within a single project and organization, and the modules were designed to work in that context, and only in that context. Nonetheless, it is instructive to describe some of the many challenges encountered because it should be immediately apparent how much more severe they would have been if the system was to have been composable across projects and work organizations.

- Syntax: Syntactical problems were relatively simple in that everything was done in one of two languages $\mathrm{C}$, or a high-level language called RANDAbel that was used by the decision models. The same underlying data bases were used for such things as geography, terrain, country names, and so on. It was therefore painful but relatively straightforward to assure that the various components of the RSAS would work together-i.e., the simulation would run.

- Semantics: Semantic issues were a major focus of attention by designers and analysts. Labeling factors affecting decisions so as to be meaningful and intuitive was critical, and was strongly guided by theory (e.g., strategic theory's concept of levels of conflict in an escalation ladder). The meaning associated with each value of such factors had to be 
"defined," usually in prose with examples, and communicated effectively to all developers. Similarly, the building-block operations had to be understood. If such an operation were to "Fall back to the such-and-such river line," then it was necessary for all users to have the same conception of what that meant. This was nontrivial and could not be accomplished with full rigor, but within the single project, it could be made to work well.

- Pragmatics: Issues of pragmatics were greatly mitigated because of the single-project environment, but were initially substantial. For example, a concept of "escalate" had to be understood in context: it meant taking actions that would change the nature of conflict to that associated with the next rung of the escalation ladder-as the ladder had been defined in the project. If an outsider had interpreted the word "escalate," he might well have thought in terms of a larger escalation (skipping one or more levels), or he might have thought in terms of something in between two of the allowed levels. To use another example, the concept of "exploit breakthrough" could only be understood correctly by knowing the nature of the simulation model and the way it attempted to reflect the consequences (not the detailed dynamics) of breakthrough operations.

- Assumptions: There were many tacit assumptions built into the RSAS and its many components. Although substantial efforts were made to write down the assumptions and rationale, this could only go so far. For example, a building-block option might use the current value of the FLOT position in a sector; implicitly, however, the option was assuming that Red forces were exclusively on one side of the FLOT and that Blue forces were exclusively on the other. That was quite reasonable given the nature of the simulation, and even the nature of war as envisioned at the time, but it was most certainly a major hidden assumption.

- Validity: Issues of validity are always present in models, but in the RSAS there were special issues. For example, if someone built a war plan that he regarded as better or more interesting than the ones already available, that plan could be "plugged in" and run as a black box. However, it might or might not correspond to something that could be followed in the real world. For example, such a plan might have exploited some weakness in the combat simulation by using tactics that would prove superior in the simulation but would, in the real world, be ruled out by factors not explicitly in the model. Although this particular problem did not actually exist in the RSAS, suppose that the combat simulation did not penalize a side for not maintaining some tactical and operational reserves. A player could then "cheat" by putting all forces on line, achieving a high force ratio, and breaking through-even though, in the real world, such a tactic would be rare and would often be severely punished by flanking attacks into the unprotected rear.

If we now turn to the political decision models, there were many interesting examples of the composability-related issues. One such example arose in a simulation of war in Europe. For the particular set of assumed initial conditions, Blue found itself losing, so it followed NATO doctrine by launching a "demonstrative nuclear strike" to reestablish deterrence in the jargon on NATO thinking. Red, however, saw the resulting level of conflict to be "limited nuclear war"; when it responded in what it thought of as "in kind," it was actually escalating again. Blue saw the result as nuclear war fighting, and countered. Depending on details of the initial conditions and other factors, Blue or Red escalated to general nuclear war. All of this occurred "by accident." From a computer-science perspective, it occurred because of a mismatch in how the two agents interpreted the underlying meaning of the other side's escalation. That might be regarded as an error in semantics. However, it also mirrored precisely the kind of misunderstanding that theorists, generals, and political leaders worried about in the real world. Indeed, even if the sides had the same escalation ladders and the same conceptual meaning for the rungs, should the two sides both evaluate the new level of conflict with the same rules (e.g., fewer than five nuclear weapons would mean "demonstrative")? That would be unrealistic to assume. However, it would tidy up the simulation.

This example from two decades ago has shown how deep and subtle problems can arise when attempting to "compose" models from modules. The difficulties are not software problems, but rather something very different. The challenges can be mitigated by careful decomposition into modules via MRM, with considerable discipline in defining and documenting related concepts substantively, by using graphical methods to help summarize and communicate relationships, and by having short substantive descriptions of module assumptions and assumptions, as well as their inputs and outputs.

Were a new RSAS to be developed today, twenty years later, it could benefit from and be more composable as the result of using modern and emerging technologies such as envisioned by Pohl (2005) and applied to conceptual modeling by Tolk and Turnitsa (2007):

- The meaning of concepts, including possible multi-faceted interpretations, can be captured in a data dictionary (which the RSAS had) and structured using taxonomies and thesauri. 


\section{Davis and Tolk}

- Associations and abstraction structures can be captured in ontological structures. New graphical interfaces will allow visualizing such ontologies in a way that they are understandable to all participating experts. Some examples of related work are described by Dobrev (2006). Underlying concepts and application examples are described by Hayes et al. (2005).

- As a last example for this paper, OWL for services (OWL-S) supports the selection and orchestration of services with a service profile providing a concise description of the capabilities implemented by the service (What it does), their service model describing the behavior and state-changes of a service (How it works) based on formal specifications of inputs, outputs, preconditions, and effects (IOPE), and their service grounding defining how to make use of a service (How to access it). The how-it-works description, however, is for the "public view" of the model and seldom describes fully the underlying assumptions. It is a research issue to find out how to do better.

We should emphasize the principal challenges remain at the modeling level-understanding conceptually the components and their alignment in a composition. The technologies and methods can support the process and even build a frame enabling the use of intelligent software agents to help the composition process, but the conceptual challenges cannot be solved by the technology. Nonetheless, choosing the right technology can help materially.

\subsection{Counterterrorism Models}

As a current example of modeling in which the issues we have talked about arise, we can mention ongoing RAND work by Davis, Hillestad, and Gvineria to build counter terrorism models for policy analysis. Suppose that a counter-terrorism model has the concept of a population's sympathy for the terrorist cause. That degree of sympathy may be seen as depending on the attractiveness of the cause and organization, and the attractiveness of its use of terrorism; the costs perceived in supporting the cause; and the ability of the population to tolerate more pain. Each of these can be decomposed into contributing factors, which can in turn be further decomposed. The result is a more-orless hierarchical tree, but with cross-branch interactions. Note that even if the hierarchical tree is a reasonable approximation, the sympathy component interacts with other portions of the model. The model as a whole can be thought of as a system-dynamic model, including feedback loops and interacting processes.

As different people work on the various components of this model, it is clearly necessary for them to have a similar understanding of what the concepts mean, how they are defined, how they are measured, what the values of the measurement scale mean, and so on. For example, sympathy for the political Islamism might be a subcomponent of attractiveness. What does more or less sympathy mean? In describing a population as very sympathetic, would we be implying that the population believed passionately in instituting Sharia law? What if the population were supportive because the Islamist movement included numerous social services and a higher degree of integrity than other factions provide? Which would raise the level of sympathy more: an ardent belief that the only constructive organization was the Islamist one, or an ardent belief in, say, Wahabbi Islam? The point here is that the concept of sympathy--along with many other concepts in social science is multifaceted; it is only for shortcut purposes that we lump them together. There is no single-dimensional scale on which to measure sympathy rigorously. However, for the purposes of modeling and analysis it might be very useful to adopt such a simplifying scale.

The reader can probably appreciate immediately that it would be straightforward to assure that various components of sympathy could be combined in a model-perhaps using linear weighted sum of factors. The syntactical problems, and even fuzzily defined meanings, could be handled. The factors could be put on the same scale so that combining them were meaningful and in some sense logically consistent with a plausible (but highly simplified theory). This, however, would be naïve, since the various factors actually combine in a number of different ways as described in a forthcoming RAND report on the modeling. It is not yet easy to say, for example, "If you can think of an additional factor contributing to sympathy, code it up, and we'll connect the model in." How that model would connect substantively would depend, for example, on whether the factor in question was an independent contributor to sympathy, a potential substitute for another, an "enhancer" or "depressor" of other factors, and so on.

Consider next that building a model of enthusiasm for political Islam would probably do things very differently if motivated by experiences in Palestine rather than, say, Iran, Iraq, or Saudi Arabia. Many of the differences might be only tacit. For example, the model might be conceived in a construct in which religion and the Islamist concept of unifying state, religion, and culture were fundamental. A different model, with the same explicit factors might be conceived around the "practical" aspects of Islamism (e.g., social services) and the idealistic aspects (religion), but not so much issues of law and culture.

Our point with the example is by no means to suggest that such modeling cannot be done, but rather that the idealized vision of composability will be unlikely to apply. It is possible for the integrative modeler to take the ideas and perhaps even the algorithms of other workers worldwide and fit them into a composite model, but to imagine that 
this could be done by doing a simple web search, downloading components that might be found on the web, and then snapping them together, could not easily be more wrong-headed. The question is how much work would be required to make use of the various would-be components, which would almost surely be heterogeneous. Clearly, the technology that we discussed in the previous example could help. A challenge is to see what would help most and to get those tools working.

\section{CONCLUSIONS}

The M\&S community has made significant steps towards composable M\&S services. In particular, this includes application of model-based data engineering as first proposed for M\&S only a few years ago (Tolk 2003) and a is now even featured in a textbook (Zeigler and Hammond 2007). Such efforts can materially support professional development of operational M\&S services to make visions of intelligent software systems as defined by Pohl (2005) possible. The ontological research conducted in support of the semantic web is supporting these goals as well and are likely to contribute new methods in support of composable solutions in multi-resolution domains.

Despite these significant developments on the technical level, the understanding of conceptual assumptions and constraints, and the alignment of describing conceptual models in support of a common conceptual flow in the resulting system of systems, remains the pivotal element for success. While new technical development can support the analyst, this task remains his responsibility. Understanding this challenge and promoting broadly applicable solutions is the next grand challenge to support composable solutions as envisioned in the report NRC (2006).

\section{REFERENCES}

Bartholet, R. G., D. C. Brogan, P. F. Reynolds Jr., and J. C. Carnahan. 2004. In search of the philosopher's stone: simulation composability versus componentbased software design. In Proceedings of the Fall Simulation Interoperability Workshop. IEEE CS Press.

Bartholet, R. G., D. C. Brogan, and P. F. Reynolds Jr. 2005. The computational complexity of component selection in simulation reuse. Proceedings of the Winter Simulation Conference, IEEE CS Press.

Benjamin, P., M. Patki, and R. Mayer. 2006. Using ontologies for simulation modeling. In Proceedings of the Winter Simulation Conference, ed. L. F. Perrone, F. P. Wieland, J. Liu, B. G. Lawson, D. M. Nicol, and R. M. Fujimoto, 1151-1159. Piscataway, New Jersey: Institute of Electrical and Electronics Engineers, Inc.

Bigelow, J. H., and P. K. Davis. 2003. Implications for model validation of multi-resolution, multi-perspective modeling (MRMPM) and exploratory analysis. RAND.

Daconta, M., L. Obrst, and K. Smith. 2003. The semantic Web: the future of XML, Web services, and knowledge management. John Wiley, Inc.

Davis, P. K. 1989. Some lessons learned from the building of red agents in the RAND strategy assessment system. RAND N-3003-OSD.

Davis, P. K. 2002. Analytic architecture for capabilitiesbased planning, mission-system analysis, and transformation. RAND MR-1513-OSD.

Davis, P. K., and R. H. Anderson. 2003. Improving the composability of Department of Defense models and simulations. RAND MG-101-OSD.

Davis, P. K., and J. H. Bigelow. 1998. Experiments in MRM. RAND MR-100-DARPA.

Davis, P. K., and R. Hillestad. 1993. Families of models that cross levels of resolution: issues for design, calibration and management. In Proceedings of the Winter Simulation Conference. Piscataway, New Jersey: Institute for Electrical and Electronics Engineers, Inc.

Davis, P. K., and R. K. Huber. 1992. Variable-resolution combat modeling: motivations, issues, and principles. RAND Notes.

Davis, P. K., J. H. Bigelow, and J. McEver. 2000. Informing and calibrating a multi-resolution exploratory analysis model with high resolution simulation: the interdiction problem as a case history. In Proceedings of the Winter Simulation Conference, ed. J. A. Joines, R. R. Barton, K. Kang, P. A. Fishwick, 316-325. Piscataway, New Jersey: Institute for Electrical and Electronics Engineers, Inc.

Dobrev, P. 2006. Knowledge interoperability for the semantic Web applications. In John Vincent Atanasoff Information Days, Proceedings of the Young Researchers Session, ed. Galia Angelova et al., 20-26.

Gruber, G. R. 1995. Toward principles for the design of ontologies used in knowledge sharing. International Journal of Human Computer Studies 45: 907-928.

Hayes, P., T. C. Eskridge, R. Saavedra, T. Reichherzer, M. Mehrotra, and D. Bobrovnikoff. 2005. Collaborative knowledge capture in ontologies. ACM Proceedings KCap'05 - 3rd Int. Conf. on Knowledge Capture. ACM Press.

Hofmann, M. 2004. Challenges of model interoperation in military simulations. Simulation 80:659-667.

National Research Council. 2006. Defense modeling, simulation, and analysis: meeting the challenge. Committee on Modeling and Simulation for Defense Transformation. National Academies Press.

Page, E. H., and J. M. Opper. 1999. Observations on the complexity of composable simulation. In Proceedings of the Winter Simulation Conference, ed. P. A. Farrington, H. B. Nembhard, D. T. Sturrock, G. W. Ev- 
ans, 553-560. Piscataway, New Jersey: Institute for Electrical and Electronics Engineers, Inc.

Page, E. H., R. Briggs, and J. A. Tufarolo. 2004. Toward a family of maturity models for the simulation interconnection problem. Proceedings Spring Simulation Interoperability Workshop, IEEE CS Press.

Perme, D., S. Diallo, C. Pandolfo, B. Tedesco, A. Tolk, and H. Tran. 2007. Joint event data initialization services (JEDIS) - implementing a service oriented architecture for initialization. Proceedings Spring Simulation Interoperability Workshop, IEEE CS Press.

Petty, M. D., and E. W. Weisel. 2003. A composability lexicon. In Proceedings of the Spring Simulation Interoperability Workshop. IEEE CS Press.

Pohl, J. 2005. Intelligent software systems in historical context. In Intelligent Decision Support Systems in Agent-Mediated Environments, ed. G. E. PhillipsWren and L. C. Jain. IOS Press.

Pullen, J. M., R. Brunton, D. Brutzman, D. Drake, M. R. Hieb, K. L. Morse, and A. Tolk. 2004. Using Web services to integrate heterogeneous simulations in a grid environment. In Computational Science - ICCS 2004: 4th International Conference, Kraków, Poland, June 6-9, Proceedings, Part III, Series: Lecture Notes in Computer Science, 835 - 847. Heidelberg: SpringerVerlag.

Radharamanan, R., and P. A. Wilsey. 1999. Ruminations on the implications of MRM on DIS/HLA. DIS-RT, p. 101, 3rd International Workshop on Distributed Interactive Simulation and Real-Time Applications.

Reynolds Jr., P. F., A. Natrajan, and S. Srinivasan. 1997. Consistency maintenance in multi-resolution simulations. ACM Transactions on Modeling and Computer Simulation, 7(3): 368-392.

Schwabe, W. 1990. Analytic war plans: adaptive forceemployment logic in the RAND strategy assessment system (RSAS). RAND.

Silver, G. A., L. W. Lacy, and J. A. Miller. 2006. Ontology based representations of simulation models following the process interaction world view. In Proceedings of the Winter Simulation Conference, ed. L. F. Perrone, F. P. Wieland, J. Liu, B. G. Lawson, D. M. Nicol, and R. M. Fujimoto, 1168-1176. Piscataway, New Jersey: Institute of Electrical and Electronics Engineers, Inc.

Spiegel, M., P. F. Reynolds, and D. Brogan. 2005. A case study of model context for simulation composability and reusability. In Proceedings of the Winter Simulation Conference, ed. M. E. Kuhl, N. M. Steiger, F. B. Armstrong, and J. A. Joines, 437-444. Piscataway, New Jersey: Institute of Electrical and Electronics Engineers, Inc.

Tolk, A. 1999. Non-monotonicities in HLA-federations. In Proceedings of the Spring Simulation Interoperability Workshop. IEEE CS Press
Tolk, A. 2003. Common data administration, data management, and data alignment as a necessary requirement for coupling C4ISR systems and M\&S. Journal for Information and Security 12(2): 164-174.

Tolk, A. and S. Y. Diallo. 2005. Model-based data engineering for Web services. IEEE Internet Computing 9(4): 65-70.

Tolk, A., and C. D. Turnitsa. 2007. Conceptual modeling of information exchange requirements based on ontological means. In Proceedings of the Winter Simulation Conference, ed. S. G. Henderson, B. Biller, M.-H. Hsieh, J. Shortle, J. D. Tew, and R. R. Baron. Piscataway, New Jersey: Institute of Electrical and Electronics Engineers, Inc.

Tolk, A., Diallo, S.Y., Turnitsa, C.D., and Winters, L.S. 2006. Composable M\&S Web services for net-centric applications. Journal for Defense Modeling \& Simulation 3(1): 27-44

Turnitsa, C. D., and A. Tolk. 2007. Federated ontologies supporting a merged worldview for distributed systems. In Proceedings of the AAAI 2007 Fall Symposia.

Weisel, E. W., M. D. Petty, and R. R. Mielke. 2003. Validity of models and classes of models in semantic composability. In Proceedings of the Fall Simulation Interoperability Workshop. IEEE CS Press.

Yilmaz L. and A. Tolk. 2007. A unifying multi-model taxonomy and architecture for agent-based decisionsupport. In Intelligent Decision Making: An AI-based Approach, ed. Phillips-Wren, Ichalkaranje, and Lakhmi. Springer-Verlag.

Yilmaz, L. 2004. On the need for contextualized introspective simulation models to improve reuse and composability of defense simulations. Journal of Defense Modeling and Simulation 1(3): 135-145.

Yilmaz, L. and T. I. Oren. 2004. A conceptual model for reusable simulations within a model-simulator-context framework. In Proceedings of the Conference on Conceptual Modeling and Simulation, Genoa, Italy.

Zeigler, B. P., and P. E. Hammonds. 2007. Modeling and simulation-based data engineering: introducing pragmatics into ontologies for net-centric information exchange. Academic Press.

Zeigler, B. P., H. Praehofer, and T. G. Kim. 2000. Theory of modeling and simulation. 2nd ed. Academic Press.

\section{AUTHOR BIOGRAPHIES}

PAUL K. DAVIS is a Principal Researcher at the RAND Corporation, and a Professor of Policy Analysis at the Pardee RAND Graduate School. He has a B.S. from the University of Michigan and a Ph.D. in chemical physics from MIT. Dr. Davis has recent books deal with capabilitiesbased planning, portfolio analysis in defense planning, effects-based planning, strategy for the influence component of counterterrorism, and advanced methods of modeling 
and simulation. His e-mail address is <pdavisarand. org $>$.

ANDREAS TOLK is Associate Professor for Engineering Management and Systems Engineering of Old Dominion University's Modeling, Simulation, and Visualization Faculty. He is also a Senior Research Scientist at the Virginia Modeling Analysis and Simulation Center (VMASC). He holds a M.S. in Computer Science (1988) and a Ph.D. in Computer Science and Applied Operations Research (1995), both from the University of the Federal Armed Forces of Germany in Munich. He is a member of SCS and SISO. His e-mail address is <atolkeodu. edu>. 